\title{
An improved model for the parallel hot wire: application to thermal conductivity measurement of low density insulating materials at high temperature
}

\author{
Yves Jannot $^{1^{*}}$, Alain Degiovanni ${ }^{1,2}$ \\ ${ }^{1}$ Université de Lorraine, CNRS, LEMTA, F-54500 Vandœuvre-lès-Nancy, France \\ ${ }^{2}$ Université Internationale de Rabat, Pôle Energie, LERMA, Rocade Rabat-Salé, 11100, Sala \\ Al Jadida, Morocco
}

\begin{abstract}
This paper presents a new model of the the transient temperature at a point situated at a distance $d$ of the heating wire, in a parallel hot wire measurement device. A preliminary theoretical study shows the limits of the estimation method proposed by the standard ISO8894-2. First, we proposed an optimal processing method, based on the model used in the standard, to improve the estimation of the thermal conductivity. Then, a new model based on the quadrupolar formalism is developed, it takes into account: the mass and the radius of the heating wire, the thermal contact resistance between the heating wire and the sample and the mass of the thermocouple. A theoretical study shows that this model enables a precise estimation of the thermal conductivity of a large range of materials and that it makes also possible to obtain an estimation of the volume heat capacity. An experimental study has been realized using a very low density material (polystyrene with $\rho=15 \mathrm{~kg} \mathrm{~m}^{-3}$ ) at ambient temperature and a reference material (Silcal 1100) at temperatures varying from $200^{\circ} \mathrm{C}$ to $1000^{\circ} \mathrm{C}$ to validate the results of the theoretical study. Compared to known thermal properties of these two materials, the thermal conductivity was estimated with a deviation lower than 3.4 $\%$ and the volume heat capacity was estimated with a deviation lower than $10 \%$.
\end{abstract}

\section{Introduction}

A variant of the hot wire method consists in placing in the medium to characterize a thermocouple parallel to the heating wire and to record its temperature. The measurement of this temperature makes it possible to estimate the thermal conductivity and the thermal diffusivity of the medium. This transient method called the parallel hot wire method was the object of the standard ISO 8894-2 [1] concerning the determination of the thermal conductivity of refractory materials.

The device of the parallel hot wire associated with the estimation method defined by the standard was used to characterize various types of materials among them: refractories [2-3], polymers [4], soils [5] and insulating materials [6].

\footnotetext{
Corresponding author. Tel: + 333727443 08. E-mail address : yves.jannot@univlorraine.fr (Y. Jannot)
} 
The model used by the standard is based on the simplifying hypothesis that the radius of the heating wire is null. Hakansson et al [7] took into account the variation of the supply power that may be negligible if the temperature coefficient of the electrical resistance is low. Pettersson [8] studied the influence of the thermal conductivity of the wire and concluded that it has no influence on the estimation of the thermal conductivity. Grazzini et al [9] proposed a model taking into account the heating wire radius and the thermal contact resistance, leading to an analytical expression of Laplace transform $\theta_{t}(p)$ of the temperature rise $T_{t}(t)$ of the thermocouple. Nevertheless, some approximations are necessary to obtain an analytical expression of $T_{t}(t)$ and these authors conclude that their model does not provide better estimation of the thermal conductivity than the simplified model (heating wire with a null radius).

Furthermore, one can notice that the thermal capacity of the thermocouple was never taken into account in all the previously cited models.

Some studies based on numerical simulations have also been realized to estimate the time limit for the validity of the semi-infinite medium hypothesis (always considered in the hot wire methods) [10], [11], [12].

The aim of this paper is to propose a more complete quadrupolar analytical model and to estimate the error caused by the simplified model used in the standard, for various types of materials. The presented model takes into account the radius and the thermal capacity of the heating wire, the thermal contact resistances between the heating wire and the sample on one hand and between the thermocouple and the sample on the other hand, as well as the thermal capacity of the thermocouple. It will be shown that the analysis of the residues of estimation makes it possible to determine easily the time limit of validity of the hypothesis of the semiinfinite medium. An experimental study realized on two reference materials will validate the results of the theoretical study

\section{Models}

The schematic diagram of the method is presented in figure 1.

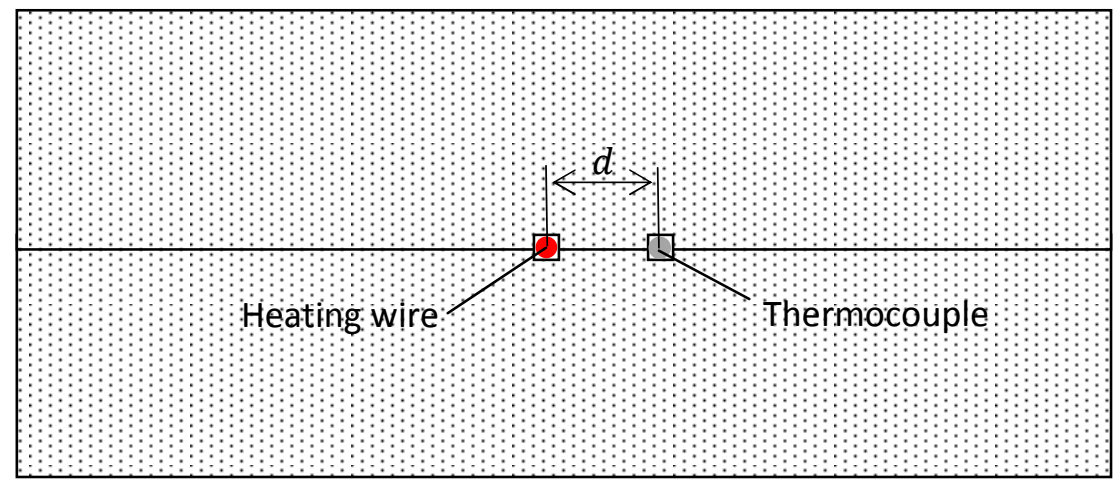

Figure 1: Schematic diagram of the parallel hot wire method 
A resistive wire with a radius $r_{w}$ is inserted in a groove realized on the surface of the bottom sample. A thermocouple is inserted in another groove realized at the surface of the same sample at distance $d$ of the heating wire. A second sample with the same dimensions as the bottom sample is then placed on it.

\section{Quadrupolar model}

A quadrupolar model M1 will now be developed considering the following hypotheses:

- The samples are semi-infinite

- The heating wire and the thermal contact resistance between the wire and the samples are taken into account

- The thermocouple is not taken into account: the thermal properties of the sample and of the thermocouple are the same and the thermal contact resistances between the thermocouple and the samples are negligible.

- The sample is optically thick

One can write the following quadrupolar relation [13] between the heating wire and a point in the solid at a distance $d$ from the wire:

$$
\left[\begin{array}{l}
\theta_{w} \\
\frac{\varphi_{w}}{p}
\end{array}\right]=\left[\begin{array}{cc}
A_{w} & B_{w} \\
C_{w} & D_{w}
\end{array}\right]\left[\begin{array}{cc}
1 & R_{c} \\
0 & 1
\end{array}\right]\left[\begin{array}{cc}
A_{s} & B_{s} \\
C_{s} & D_{s}
\end{array}\right]\left[\begin{array}{c}
\theta_{d} \\
\frac{\theta_{d}}{Z}
\end{array}\right]=\left[\begin{array}{ll}
A & B \\
C & D
\end{array}\right]\left[\begin{array}{c}
\theta_{d} \\
\frac{\theta_{d}}{Z}
\end{array}\right]
$$

With:

$$
\begin{aligned}
& A_{w}=1 \\
& B_{w}=\frac{1}{2 \pi \lambda_{w} L q_{w} r_{w}} \frac{I_{0}\left(q_{w} r_{w}\right)}{I_{1}\left(q_{w} r_{w}\right)}-\frac{1}{\rho_{w} c_{w} \pi r_{w}^{2} L p} \\
& C_{w}=\rho_{w} c_{w} \pi r_{w}^{2} L p \\
& D_{w}=\frac{q_{w} r_{w}}{2} \frac{I_{0}\left(q_{w} r_{w}\right)}{I_{1}\left(q_{w} r_{w}\right)} \\
& A_{s}=q_{s} d\left[K_{1}\left(q_{s} d\right) I_{0}\left(q_{s} r_{w}\right)+K_{0}\left(q_{s} r_{w}\right) I_{1}\left(q_{s} d\right)\right] \\
& B_{s}=\frac{1}{2 \pi \lambda_{s} L}\left[K_{0}\left(q_{s} r_{w}\right) I_{0}\left(q_{s} d\right)-K_{0}\left(q_{s} d\right) I_{0}\left(q_{s} r_{w}\right)\right] \\
& C_{s}=2 \pi L \rho_{s} c_{s} r_{w} d p\left[K_{1}\left(q_{s} r_{w}\right) I_{1}\left(q_{s} d\right)-K_{1}\left(q_{s} d\right) I_{1}\left(q_{s} r_{w}\right)\right] \\
& D_{s}=q_{s} r_{w}\left[K_{1}\left(q_{s} r_{w}\right) I_{0}\left(q_{s} d\right)+K_{0}\left(q_{s} d\right) I_{1}\left(q_{s} r_{w}\right)\right] \\
& \frac{1}{Z}=2 \pi \lambda_{s} L q_{s} d \frac{K_{1}\left(q_{s} d\right)}{K_{0}\left(q_{s} d\right)} ; q_{w}=\sqrt{\frac{p}{a_{w}}} \quad ; q_{s}=\sqrt{\frac{p}{a_{s}}}
\end{aligned}
$$

Where:

$\theta_{w} \quad$ Laplace transform of the temperature rise of the wire

$\theta_{d} \quad$ Laplace transform of the temperature rise at a distance $d$ in the solid

$I_{0}, I_{1}, K_{0}, K_{1}$ modified Bessel function of the first and second kind

$\lambda_{w} \quad$ thermal conductivity of the heating wire

$\rho_{w} c_{w}$ volume heat capacity of the heating wire

$a_{w} \quad$ thermal diffusivity of the heating wire

$L \quad$ length of the heating wire

$r_{w} \quad$ radius of the heating wire 
$d \quad$ distance between the heating wire and the thermocouple

$\lambda_{s} \quad$ thermal conductivity of the sample

$\rho_{s} c_{s} \quad$ volume heat capacity of the sample

$a_{s} \quad$ thermal diffusivity of the sample

$R_{c} \quad$ thermal contact resistance between the heating wire and the sample

$p \quad$ Laplace parameter

$\varphi_{w} \quad$ Heat flow rate in the heating wire

Then:

$\frac{\varphi_{w}}{p}=\left(C+\frac{D}{Z}\right) \theta_{d}$

And finally: $\theta_{d}(p)=\frac{\frac{\varphi_{w}}{p}}{C+\frac{D}{Z}}$

with:

$C=A_{s} C_{w}+C_{s}\left(C_{w} R_{c}+D_{w}\right)$

$D=B_{s} C_{w}+D_{s}\left(C_{w} R_{c}+D_{w}\right)$

If the thermal resistance of the heating wire can be neglected, relation (3) becomes:

$\theta_{d}(p)=\frac{\frac{\varphi_{w}}{p L} K_{0}\left(q_{s} d\right)}{\rho_{w} c_{w} \pi r_{w}^{2} p K_{0}\left(q_{s} r_{w}\right)+2 \pi \lambda q_{s} r_{w}\left[1+\rho_{w} c_{w} \pi r_{w}^{2} p R_{c} L\right] K_{1}\left(q_{s} r_{w}\right)}$

Relation (6) is identical to the one given by Grazzini et al [9].

Case where the thermocouple properties are very different from those of the samples

In this case, one can no longer consider that the thermocouple's properties are identical to those of the material to characterize. If we also consider the simplifying hypothesis that the temperature field in the sample close to the thermocouple is not modified by the thermocouple, the Laplace transform $\theta_{t}(p)$ of the temperature rise $T_{t}(t)$ at a distance $d$ from the heating wire can always be calculated using relation (3).

Since the thermocouple does not produce any heat, if $\theta_{t}(p)$ is the Laplace transform of the mean temperature of the thermocouple, one can write [12]:

$\left[\begin{array}{c}\theta_{t} \\ 0\end{array}\right]=\left[\begin{array}{cc}A_{t} & B_{t} \\ C_{t} & D_{t}\end{array}\right]\left[\begin{array}{cc}1 & R_{t} \\ 0 & 1\end{array}\right]\left[\begin{array}{c}\theta_{d} \\ \Phi_{\mathrm{d}}\end{array}\right]$

Where:

$\left[\begin{array}{ll}A_{t} & B_{t} \\ C_{t} & D_{t}\end{array}\right]$ is the quadrupolar matrix of the thermocouple

$\Phi_{\mathrm{d}}$ is the Laplace transform of the heat flow rate at a distance $d$ from the heating wire.

$R_{t}$ is the thermal contact resistance between the thermocouple and the sample. 
Neglecting the thermal resistance of the thermocouple material compared to the thermal contact resistance between the thermocouple and the sample (hypothesis of uniform temperature), one can write:

$A_{t}=1 ; B_{t}=0 ; C_{t}=\rho_{t} c_{t} \pi r_{t}^{2} L p ; D_{t}=1$

Where $r_{t}$ is the radius of the thermocouple, $L$ is its length, $\rho_{t}$ is its density and $c_{t}$ its specific heat.

Then, the relation (7) leads to the model that we will call M2:

$\theta_{t}(p)=\frac{\theta_{d}(p)}{1+C_{t} p}$

Where $C_{t}=\rho_{t} c_{t} \pi r_{t}^{2} L R_{t}$ can be considered as a time constant (s).

\section{Model used in the standard ISO 8894-2}

The model used in the standard ISO 8894-2 (that we will call M0) is an approximation calculated for a heating wire having a null radius $\left(r_{w}=0\right)$. One can use the limited developments of the Bessel functions in the vicinity of zero:

$K_{0}(x) \approx-\ln (x) ; K_{1}(x) \approx \frac{1}{x} ; I_{0}(x) \approx 1 ; I_{1}(x) \approx \frac{x}{2}$

Hence:

$A_{w} \approx 1$

$B_{w} \approx 0$

$C_{w} \approx 0$

$D_{w} \approx 1$

Considering these approximations:

$C=A_{s} C_{w}+C_{s}\left(C_{w} R_{c}+D_{w}\right)=C_{s}$

and:

$D=B_{s} C_{w}+D_{s}\left(C_{w} R_{c}+D_{w}\right)=D_{s}$

Thus:

$C_{s}=2 \pi L \rho_{s} c_{s} r_{w} d p\left[K_{1}\left(q_{s} r_{w}\right) I_{1}\left(q_{s} d\right)-K_{1}\left(q_{s} d\right) I_{1}\left(q_{s} r_{w}\right)\right] \approx 2 \pi L \sqrt{\lambda_{s} \rho_{s} c_{s}} d \sqrt{p} I_{1}\left(q_{s} d\right)$

$D_{s}=q_{s} r_{w}\left[K_{1}\left(q_{s} r_{w}\right) I_{0}\left(q_{s} d\right)+K_{0}\left(q_{s} d\right) I_{1}\left(q_{s} r_{w}\right)\right]=I_{0}\left(q_{s} d\right)$

So that:

$C+\frac{D}{Z}=2 \pi L \sqrt{\lambda_{s} \rho_{s} c_{s}} d \sqrt{p} I_{1}\left(q_{s} d\right)+2 \pi \lambda L q d \frac{K_{1}\left(q_{s} d\right)}{K_{0}\left(q_{s} d\right)} I_{0}\left(q_{s} d\right)$ 
$C+\frac{D}{Z}=2 \pi L \sqrt{\lambda_{s} \rho_{s} c_{s}} d \sqrt{p}\left[\frac{I_{1}\left(q_{s} d\right) K_{0}\left(q_{s} d\right)+K_{1}\left(q_{s} d\right) I_{0}\left(q_{s} d\right)}{K_{0}\left(q_{s} d\right)}\right]=\frac{2 \pi \lambda L}{K_{0}\left(q_{s} d\right)}$

We have: $\theta_{t}(p)=\frac{\varphi_{w}}{p} \frac{K_{0}\left(q_{s} d\right)}{2 \pi \lambda_{s} L}$

According to Carslaw and Jaeger [14]:

$L^{-1}\left[\frac{1}{p} K_{0}(x \sqrt{p})\right]=\frac{1}{2} \int_{\frac{x^{2}}{4 t}}^{\infty} \frac{e^{-u}}{u} d u$

with: $-E_{i}(y)=\int_{y}^{\infty} \frac{e^{-u}}{u} d u$

Thus: $T_{t}(t)=-\frac{\varphi_{w}}{4 \pi \lambda_{s} L} E_{i}\left(\frac{d^{2}}{4 a_{s} t}\right)$

Where $E_{i}$ is the exponential integral

This solution was given by Carslaw and Jaeger [14], it was deducted from the temperature field in a semi-infinite medium in which a heat flow rate step is applied on a line.

Figure 2 shows the temperature simulated using the relations (3) and (20). The value of the temperature calculated by the relation (20) (standard) is always lower than the value calculated by the quadrupolar model. The two models converge for long times. Table 1 gives the parameters values used for the simulation

Table 1: Values of the parameters used for the simulations in figure 2

\begin{tabular}{|l|l|l|l|l|l|c|c|}
\hline & $r_{w}$ & \multicolumn{1}{c|}{$\lambda_{w}$} & $\rho_{w} c_{w}$ & $d$ & $\lambda_{s}$ & $\rho_{s} c_{s}$ & $R_{c}$ \\
\hline & $\mathrm{mm}$ & $\mathrm{W} \mathrm{m}^{-1} \mathrm{~K}^{-1}$ & $\mathrm{~J} \mathrm{~m}^{-3} \mathrm{~K}^{-1}$ & $\mathrm{~mm}$ & $\mathrm{~W} \mathrm{~m}^{-1} \mathrm{~K}^{-1}$ & $\mathrm{~J} \mathrm{~m}^{-3} \mathrm{~K}^{-1}$ & $\mathrm{~K} \mathrm{~W}^{-1}$ \\
\hline Figure 2a) & 0.25 & 11.3 & $3.78 \times 10^{6}$ & 5 & 0.1 & $5 \times 10^{5}$ & 3.5 \\
\hline Figure 2b) & 0.25 & 11.3 & $3.78 \times 10^{6}$ & 5 & 0.2 & $5 \times 10^{5}$ & 3.5 \\
\hline
\end{tabular}
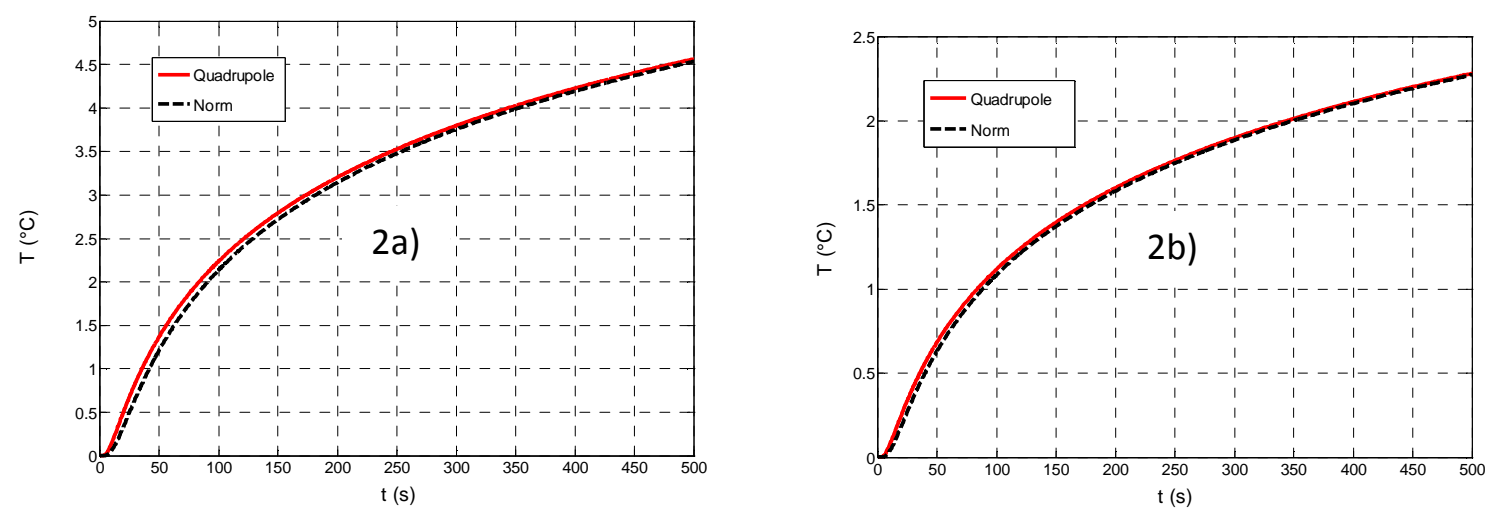

Figure 2: Temperature of the thermocouple simulated with the model of the standard and with the quadrupolar model: $2 a$ ) $\lambda_{s}=0.1 \mathrm{~W} \mathrm{~m}^{-1} \mathrm{~K}^{-1}$; 2b) $\lambda_{s}=0.2 \mathrm{~W} \mathrm{~m}^{-1} \mathrm{~K}^{-1}$

\section{Parameters estimation}

Quadrupolar models (M1 and M2) 
The thermal properties of the resistive heating wire are supposed to be known so that the unknowns are the thermal conductivity $\lambda_{s}$ and volume capacity $\rho_{s} c_{s}$ of the samples and the thermal contact resistance $R_{c}$ between the heating wire and the samples. The time constant $C_{t}$ can be added as an unknown parameter if the sample thermal properties are very different from those of the thermocouple.

The temperature rise $T_{t}(t)$ can be calculated by an inverse Laplace transform applied to $\theta_{t}(p)$ realized by using the De Hoog algorithm [15]. The Levenberg-Marquart algorithm [16] is used to find the values of the unknown parameters that minimize the sum of the quadratic errors:

$S=\sum_{n=1}^{N}\left[T_{t_{\text {exp }}}(t)-T_{t_{\text {mod }}}(t)\right]^{2}$

The parameters $\lambda_{s}, \rho_{s} c_{s}$ and $R_{c}$ must be estimated in the model M1 and the parameters $\lambda_{s}$, $\rho_{s} c_{s}, R_{c}$ and $C_{t}$ must be estimated in the model $\mathrm{M} 2$.

\section{$\underline{\text { Standard ISO 8894-2 (Model M0) }}$}

We have: $T_{t}(2 t)=-\frac{\varphi_{w}}{4 \pi \lambda_{s} L} E_{i}\left(\frac{d^{2}}{8 a_{s} t}\right)$

Thus: $U=\frac{T_{t}(2 t)}{T_{t}(t)}=\frac{E_{i}\left(\frac{d^{2}}{8 a_{s} t}\right)}{E_{i}\left(\frac{d^{2}}{4 a_{s} t}\right)}=\frac{E_{i}\left(\frac{u}{2}\right)}{E_{i}(u)}$

Where: $u=\frac{d^{2}}{4 a_{s} t}$

For each value of $u$ one can calculate $E_{i}(u)$ and $U=\frac{E_{i}\left(\frac{u}{2}\right)}{E_{i}(u)}$. Thus the function $E_{i}(u)=$ $f\left[U=\frac{E_{i}\left(\frac{u}{2}\right)}{E_{i}(u)}\right]$ can be plotted and modelled. We have identified the following polynomial function which enables its calculation with a precision better than $1 \%$ :

$$
\begin{aligned}
& E_{i}(u)=a_{0}+a_{1} U+a_{2} U^{2}+a_{3} U^{3}+a_{4} U^{4}+a_{5} U^{5}+a_{6} U^{6}+a_{7} U^{7} \\
& a_{0}=474.926168472597 \\
& a_{1}=-1574.223079517534 \\
& a_{2}=2262.705889755608 \\
& a_{3}=-1816.820318813171 \\
& a_{4}=877.286213344994 \\
& a_{5}=-254.261946376719 \\
& a_{6}=40.904415135482 \\
& a_{7}=-2.815400284900
\end{aligned}
$$

The principle of the method is the following:

- The temperature rise $T_{t}$ is recorded during a time $2 t_{f}$ 
- The ratio $U=\frac{T_{t}(2 t)}{T_{t}(t)}$ is calculated for each time value comprised in the interval $\left[0 t_{f}\right]$

- One can deduce $E_{i}\left(u=\frac{d^{2}}{4 a_{s} t}\right)$ from relation (24) then $\lambda_{s}$ is obtained using the relation:

$$
\lambda_{s}=-\frac{\varphi_{w}}{4 \pi L T_{t}(t)} E_{i}\left(\frac{d^{2}}{4 a_{s} t}\right)
$$

The calculation must be done on a time interval where the hypotheses (negligible mass of the heating wire and semi-infinite medium) are valid, we will see further how this time interval can be determined.

When applying the standard ISO 8894-2, $E_{i}(u)$ is deduced from $U=\frac{T_{t}(2 t)}{T_{t}(t)}$ by interpolation in a table of numerical values. The use of relation (24) is more convenient.

\section{Comparison of the models}

To evaluate the precision of the estimations based on the two models we have realized 2D numerical simulations of the system represented in figure 1 with COMSOL. The following boundary conditions have been considered:

- The temperature on the unheated face of the samples is uniform and constant

- The heat losses coefficient on the lateral faces of the samples is $h=20 \mathrm{~W} \mathrm{~m}^{-2} \mathrm{~K}^{-1}$.

The following properties have also been considered:

- The heating wire with a $0.5 \mathrm{~mm}$ diameter is made of Nickel Chromium 80/20.

- The thermocouple with a $0.5 \mathrm{~mm}$ diameter has the same thermal properties as the heating wire

- The heating wire and the thermocouple are placed in grooves with a $0.5 \times 0.5 \mathrm{~mm}^{2}$ square section filled with still air.

The simulations have been realized with the thermal properties given in the table 2:

Table 2: Thermal properties used for the COMSOL simulations

\begin{tabular}{|l|l|l|}
\hline Material & \multicolumn{1}{|c|}{$\lambda$} & \multicolumn{1}{c|}{$\rho \mathrm{c}$} \\
\hline & $\mathrm{W} \mathrm{m}^{-1} \mathrm{~K}^{-1}$ & $\mathrm{~J} \mathrm{~kg}^{-1} \mathrm{~m}^{-3}$ \\
\hline Air [17] & 0.026 & $1.30 \times 10^{3}$ \\
\hline NiCr 80/20 [18] & 11.3 & $3.78 \times 10^{6}$ \\
\hline Quartzel felts $\circledR[19]$ & 0.035 & $2.04 \times 10^{4}$ \\
\hline Silcal 1100 $®[20]$ & 0.12 & $2.50 \times 10^{5}$ \\
\hline LUX500® $[21]$ & 0.22 & $7.70 \times 10^{5}$ \\
\hline NorFoam $\circledR[22]$ & 0.75 & $7.00 \times 10^{5}$ \\
\hline
\end{tabular}

The COMSOL simulations have been realized using the dimensions defined by the standard: 
the sample dimensions are $200 \times 100 \times 50 \mathrm{~mm}$ and the distance between the heating wire and the thermocouple is $d=15 \mathrm{~mm}$. The standard recommends considering the values of the thermal conductivity calculated for the values of the ratio $\frac{T_{t}(2 t)}{T_{t}(t)}$ between 1.5 and 2.4 , provided that the maximum deviation from the mean value is lower than $5 \%$.

The simulated curves have been considered as numerical experiments and processed using the three estimation methods to estimate:

- The values of $\lambda_{s}, \rho_{s} c_{s}$ and $R_{c}$ with the quadrupolar model M1.

- The values of $\lambda_{s}, \rho_{s} c_{s}, R_{c}$ and $C_{t}$ with the quadrupolar model M2.

- A curve $\lambda_{s}=f(t)$ with the model M0 (standard) enabling the estimation of a mean value.

The estimation time interval for the quadrupolar models M1 and M2 has been adjusted so that the mean value of the estimation residues is null and that the residues are centered on zero. The results are presented in table 3 .

The limit of validity of the semi-infinite medium hypothesis appears very clearly on curve of residues even for the lightest material (Quartzel) when the estimation is realized by using the model M2 (cf. figure 3b). This limit cannot be defined with the same accuracy when we use the model M1 for Quartzel since the residues are oscillating around the null value (cf. figure 3a). Compared to reference values, the values of the thermal conductivity estimated with the model M2 present a maximum deviation of $2.3 \%$ for Quartzel while it reaches $12.2 \%$ with the model M1. This deviation compared with the reference values is lower than $1 \%$ with models M1 and M2 for the 3 other materials, and residues obtained with both models are identical as shown in figure $3 \mathrm{c}$ ) and $3 \mathrm{~d}$ ).

Table 3: Estimated values of the parameters from COMSOL numerical experiments with samples dimensions $200 \times 100 \times 50 \mathrm{~mm}^{3}$ and $d=15 \mathrm{~mm}$.

\begin{tabular}{|c|c|c|c|c|c|c|}
\hline & & & Quartzel & Silcal 1100 & LUX 500 & Norfoam \\
\hline \multirow{3}{*}{ M0 (Standard) } & $t_{\text {estimation }}$ & $\mathrm{s}$ & 150 & 500 & 400 & 200 \\
\cline { 2 - 7 } & $\lambda_{s}$ & $\mathrm{~W} \mathrm{~m}^{-1} \mathrm{~K}^{-1}$ & 0.027 & 0.116 & 0.219 & 0.74 \\
\hline \multirow{3}{*}{ M1 } & $\left(\lambda_{s}-\lambda_{s_{\text {ref }}}\right) / \lambda$ & $\%$ & -22.8 & -3.3 & -0.9 & -1.2 \\
\cline { 2 - 7 } & $\lambda_{s}$ & $\mathrm{~W} \mathrm{~m}^{-1} \mathrm{~K}^{-1}$ & 0.0307 & 0.119 & 0.22 & 0.75 \\
\cline { 2 - 7 } & $\left(\rho_{s} c_{s}\right.$ & $\mathrm{kJ} \mathrm{m}^{-3} \mathrm{~K}^{-1}$ & 22.2 & 253 & 775 & 704 \\
\hline \multirow{3}{*}{ M2 } & $\left.\lambda_{s_{\text {ref }}}\right) / \lambda$ & $\%$ & -12.2 & 0.8 & 0 & 0 \\
\cline { 2 - 7 } & $\lambda_{s} c_{s}$ & $\mathrm{WJ} \mathrm{m}^{-1} \mathrm{~K}^{-1}$ & 0.0358 & 0.119 & 0.221 & 0.75 \\
\cline { 2 - 7 } & $\left(\lambda_{s}-\lambda_{s_{r e f}}\right) / \lambda$ & & 20.7 & 253 & 770 & 704 \\
\hline \multirow{2}{*}{ Reference values } & $\lambda_{s_{r e f}}$ & $\mathrm{~W} \mathrm{~m}^{-1} \mathrm{~K}^{-1}$ & 0.035 & 0.12 & 0.22 & 0.75 \\
\cline { 2 - 7 } & $\left(\rho_{s} c_{s}\right)_{\text {ref }}$ & $\mathrm{kJ} \mathrm{m}^{-3} \mathrm{~K}^{-1}$ & 20.4 & 250 & 770 & 700 \\
\hline
\end{tabular}



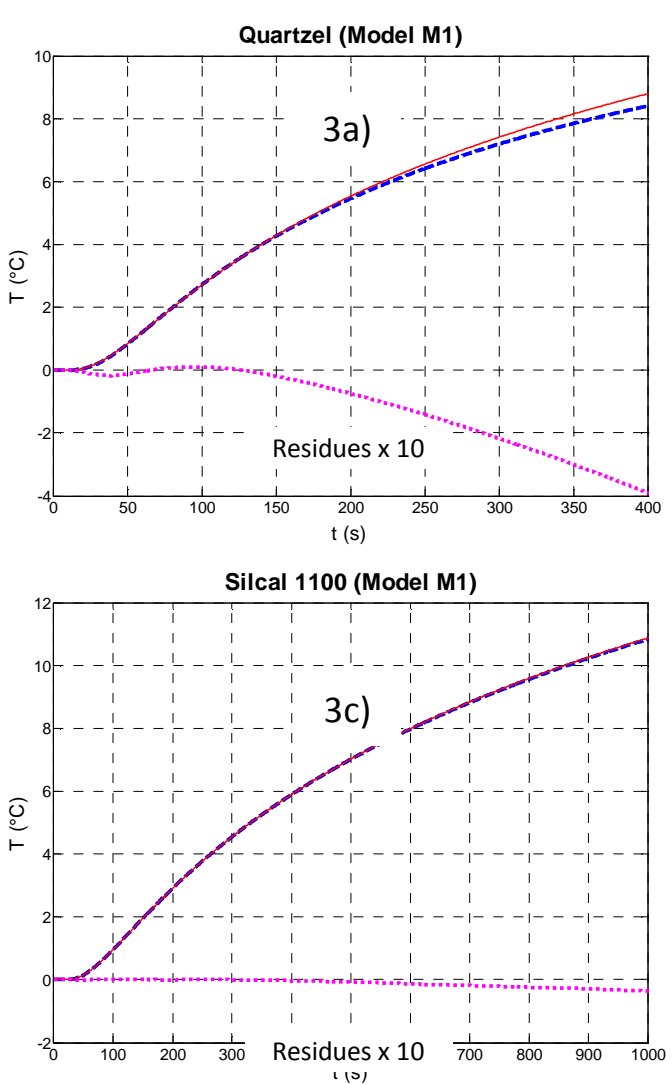
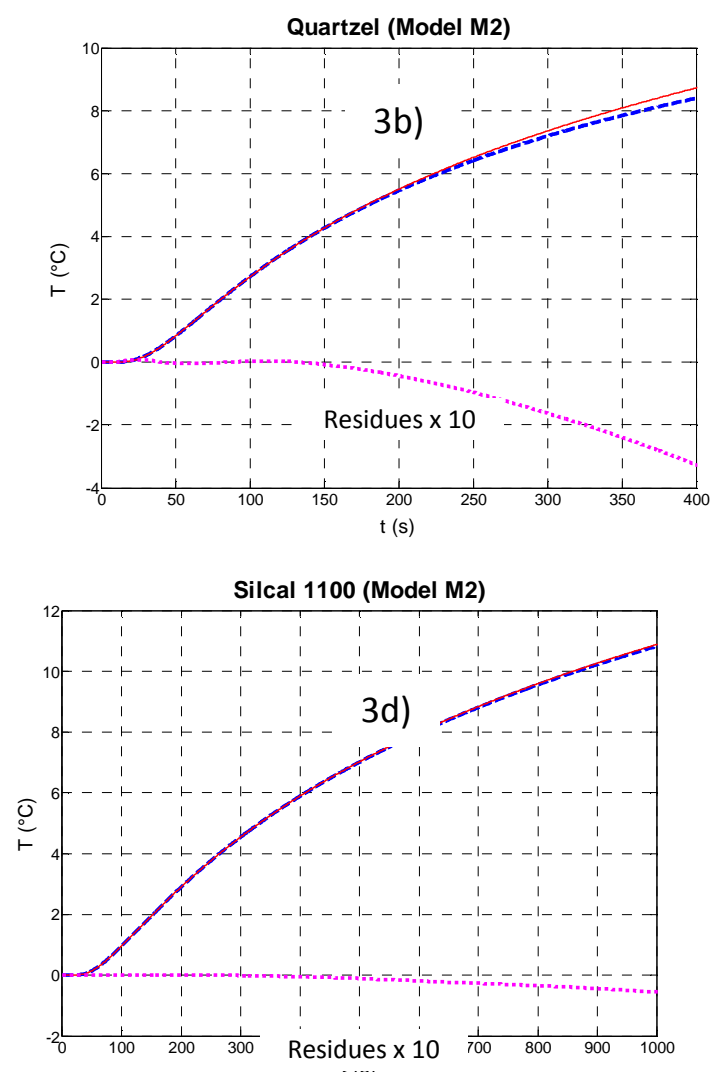

Figure 3: COMSOL simulations (--), quadrupolar model (-) and residues $\times 10$ for two materials

The application of the method of the standard leads to less precise results and always to underestimated values. The value obtained before that the hypothesis of the semi-infinite medium is no longer valid (situated in the rectangle in dotted lines on the figure 4) is more precise than the average value calculated between the values 1.5 and 2.4 of the ratio $\frac{T_{t}(2 t)}{T_{t}(t)}$ (estimation method recommended by the standard). This remark is justified by the fact that the approximation of the standard neglects the heating wire mass and radius, it is thus more precise for long times (cf. figure 2). Furthermore, figure 4 shows that the change of slope of the curve $\lambda=f\left[\frac{T_{t}(2 t)}{T_{t}(t)}\right]$ occurs in every case for $\frac{T_{t}(2 t)}{T_{t}(t)} \approx 1.6$.
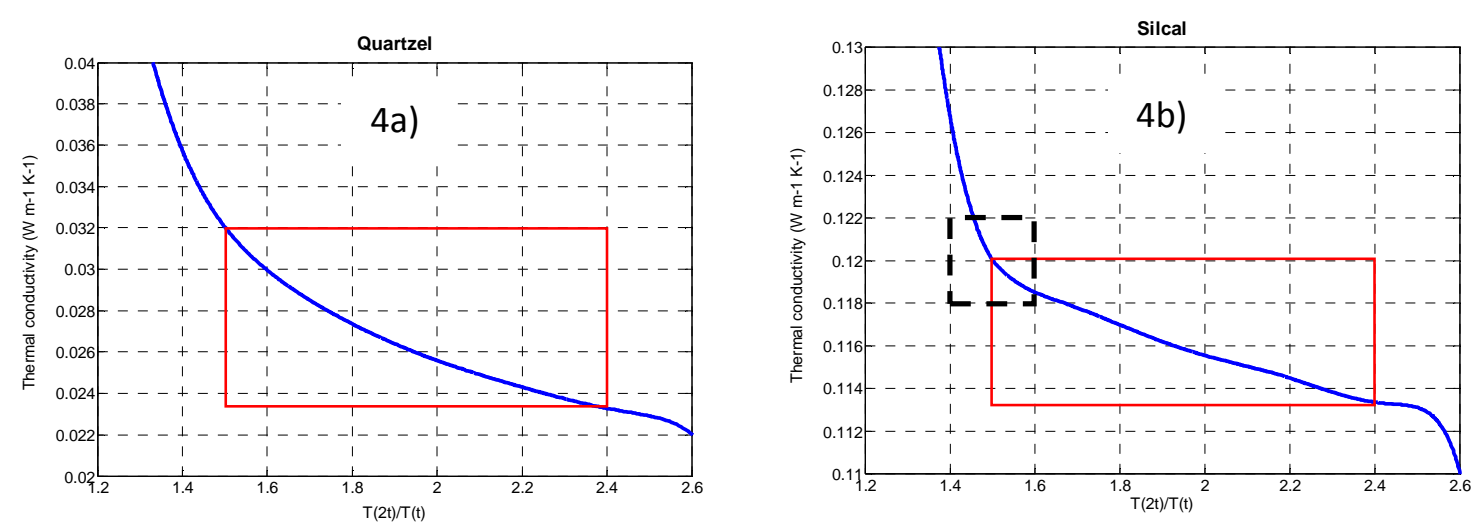

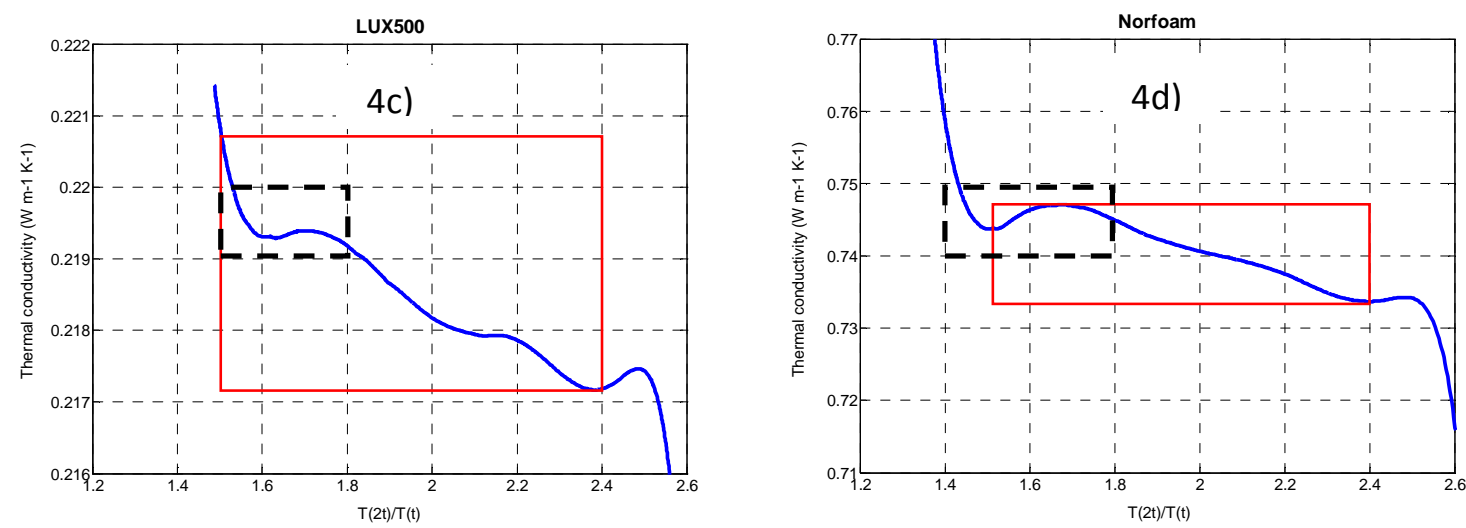

Figure 4: Thermal conductivity of the four materials estimated with the method of the standard ISO 8894-2 with sample dimensions $200 \times 100 \times 50 \mathrm{~mm}^{3}$ and $\mathrm{d}=15 \mathrm{~mm}$.

The standard method does not enable an accurate estimation of the thermal conductivity of low density materials such as Quartzel. The estimation error can reach $20 \%$ for these materials.

The standard lays down a distance $d=15 \mathrm{~mm}$ between the heating wire and the thermocouple. To test the influence of this distance on the precision of the estimation we have realized the same study but with a distance reduced to $d=5 \mathrm{~mm}$ enabling to use thinner samples; we chose a sample thickness of $30 \mathrm{~mm}$.

Figure 5 presents the values of the thermal conductivity estimated with the standard method and table 4 presents the values obtained with the three methods.

Table 4: Estimated values of the parameters from COMSOL numerical experiments with samples dimensions of $150 \times 150 \times 30 \mathrm{~mm}^{3}$ and $d=5 \mathrm{~mm}$.

\begin{tabular}{|c|c|c|c|c|c|c|}
\hline & & & Quartzel & Silcal 1100 & LUX 500 & Norfoam \\
\hline & $t_{\text {estimation }}$ & $\mathrm{s}$ & 150 & 500 & 400 & 200 \\
\hline \multirow[b]{2}{*}{ M0 (Standard) } & $\lambda_{s}$ & $\mathrm{~W} \mathrm{m^{-1 }} \mathrm{K}^{-1}$ & Undefined & 0.116 & 0.219 & 0.74 \\
\hline & $\left(\lambda_{s}-\lambda_{s_{r e f}}\right) / \lambda$ & $\%$ & - & -3.3 & -0.9 & -1.2 \\
\hline \multirow{3}{*}{ M1 } & $\lambda_{s}$ & $\mathrm{~W} \mathrm{m^{-1 }} \mathrm{K}^{-1}$ & 0.0306 & 0.120 & 0.220 & 0.750 \\
\hline & $\rho_{s} c_{S}$ & $\mathrm{~kJ} \mathrm{~m}^{-3} \mathrm{~K}^{-1}$ & 25.0 & 256 & 780 & 704 \\
\hline & $\left(\lambda_{s}-\lambda_{s_{r e f}}\right) / \lambda$ & $\%$ & -12.5 & 0 & 0 & 0 \\
\hline \multirow{3}{*}{ M2 } & $\lambda_{s}$ & $\mathrm{~W} \mathrm{~m}^{-1} \mathrm{~K}^{-1}$ & 0.0362 & 0.120 & 0.220 & 0.750 \\
\hline & $\rho_{s} c_{s}$ & $\mathrm{~kJ} \mathrm{~m}^{-3} \mathrm{~K}^{-1}$ & 20.7 & 254 & 779 & 708 \\
\hline & $\left(\lambda_{s}-\lambda_{s_{r e f}}\right) / \lambda$ & $\%$ & 3.4 & 0 & 0 & 0 \\
\hline \multirow{2}{*}{ Reference values } & $\lambda_{s_{r e f}}$ & $\mathrm{~W} \mathrm{m^{-1 }} \mathrm{K}^{-1}$ & 0.035 & 0.12 & 0.22 & 0.75 \\
\hline & $\left(\rho_{s} c_{s}\right)_{r e f}$ & $\mathrm{~kJ} \mathrm{~m}^{-3} \mathrm{~K}^{-1}$ & 20.4 & 250 & 770 & 700 \\
\hline
\end{tabular}



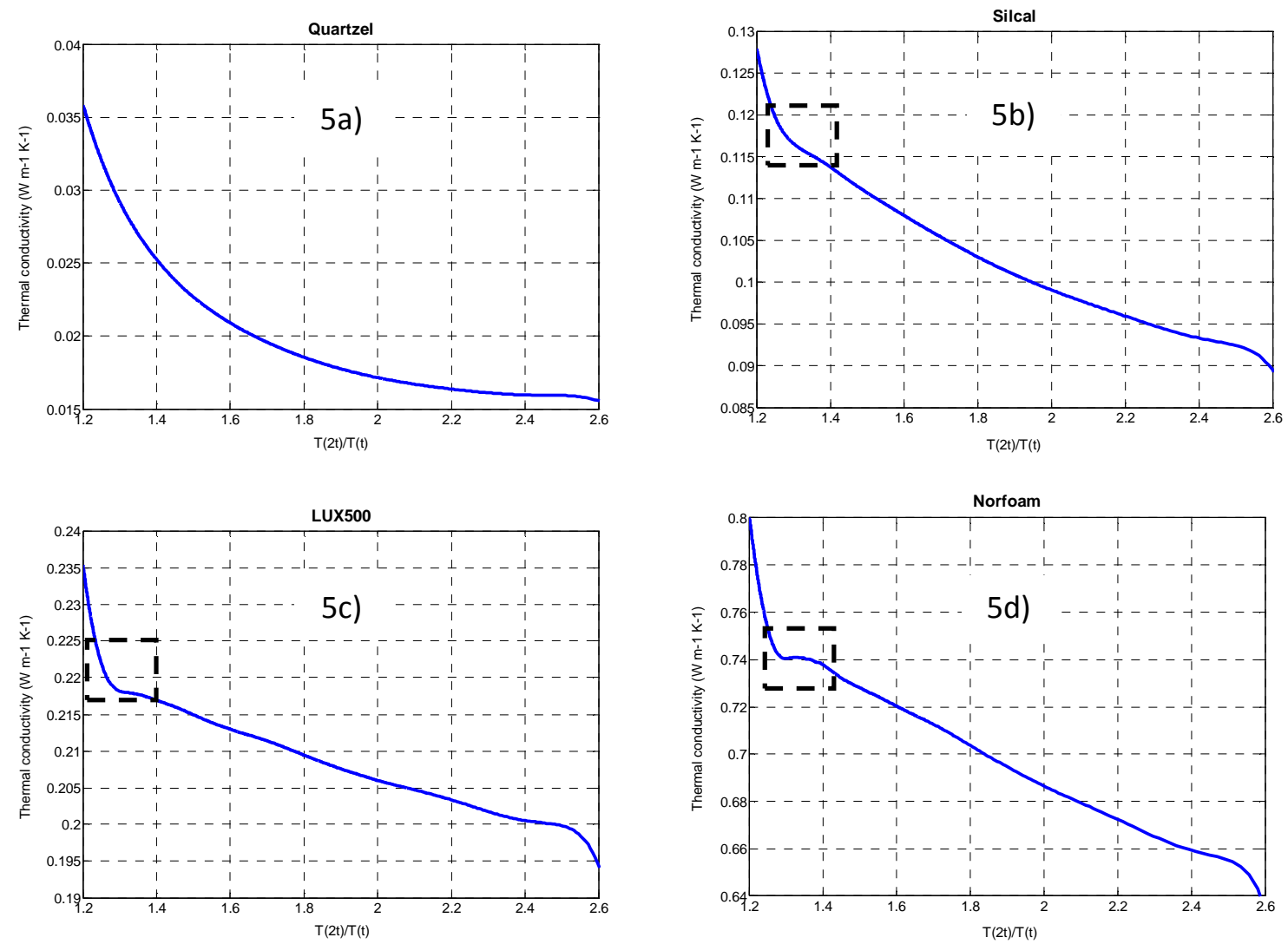

Figure 5: Values of the thermal conductivity estimated with the standard method for the four materials, with samples dimensions of $150 \times 150 \times 30 \mathrm{~mm}^{3}$ and $d=5 \mathrm{~mm}$.

One can notice that the quadrupolar model leads to the same values with $e=30 \mathrm{~mm}$ and $d=5 \mathrm{~mm}$ on one hand and with $e=50 \mathrm{~mm}$ and $d=15 \mathrm{~mm}$ on the other hand. The standard method leads to satisfying results (error less than 3\%) if the thermal conductivity is estimated at the time $t$ where the slope of the curve $\lambda=f\left[\frac{T_{t}(2 t)}{T_{t}(t)}\right]$ changes, corresponding to $\frac{T_{t}(2 t)}{T_{t}(t)} \approx 1.3$. Nevertheless, this method cannot be used to estimate a precise value of the thermal conductivity of low density materials and the slope change may appear less clearly on a noisy signal. It is remarkable that the model M2 enables the estimation of thermal conductivity of all the materials: the deviation for Quartzel (density of $26 \mathrm{~kg} \mathrm{~m}^{-3}$ ) is only $3.4 \%$ when it is $12.5 \%$ with model M1 that does not take into account the thermal capacity of the thermocouple. For all the other materials, models M1 and M2 lead to the same estimations.

\section{Materials and method}

An experimental study has been carried out to confirm these theoretical results. Two materials have been tested:

- A low density material: polystyrene with a density $\rho=15 \mathrm{~kg} \mathrm{~m}^{-3}$ to validate model M2

- A high temperature insulating material : Silcal 1100 with lower dimensions than recommended by the standard 
The dimensions of the samples of extruded polystyrene were $200 \times 100 \times 80 \mathrm{~mm}^{3}$ and the measurements have been realized at a mean temperature of $25^{\circ} \mathrm{C}$. Three experiments have been carried out.

The thermal properties of the extruded polystyrene are the following:

- Specific heat: $c=1233 \mathrm{~J} \mathrm{~kg}^{-1} \mathrm{~K}^{-1}$ at $25^{\circ} \mathrm{C}$, measured by a Setaram $\mu \mathrm{dSc} 3$ calorimeter

- Thermal conductivity: $\lambda=0.324 \mathrm{~W} \mathrm{~m}^{-1} \mathrm{~K}^{-1}$ at $25^{\circ} \mathrm{C}$, measured by a NETZCH HFM 436.

The thermal conductivity of Silcal 1100 at various temperatures has been measured by several laboratories which have established the following relation valid between 300 and 1100K [20]:

$\lambda_{\text {ref }}=0.06797+3.699 \times 10^{-5} T+6.202 \times 10^{-8} T^{2}-8.502 \times 10^{-12} T^{3}$

Where $T$ is the temperature in $\mathrm{K}$

The dimensions of the samples of Silcal were $150 \times 150 \times 30 \mathrm{~mm}^{3}$ and the measurements have been realized at $200^{\circ} \mathrm{C}, 400^{\circ} \mathrm{C}, 600^{\circ} \mathrm{C}, 800^{\circ} \mathrm{C}$ and $1000^{\circ} \mathrm{C}$. Three experiments have been carried out for each temperature.

Figure 6 presents a schematic view of the experimental setup. The samples were inserted between two stainless steel $316 \mathrm{~L}$ plates with the same cross section and with a $10 \mathrm{~mm}$ thickness. They ensure a uniform temperature on the unheated face of the samples. These plates also provide a thermal inertia that minimizes the temperature variations in the samples.

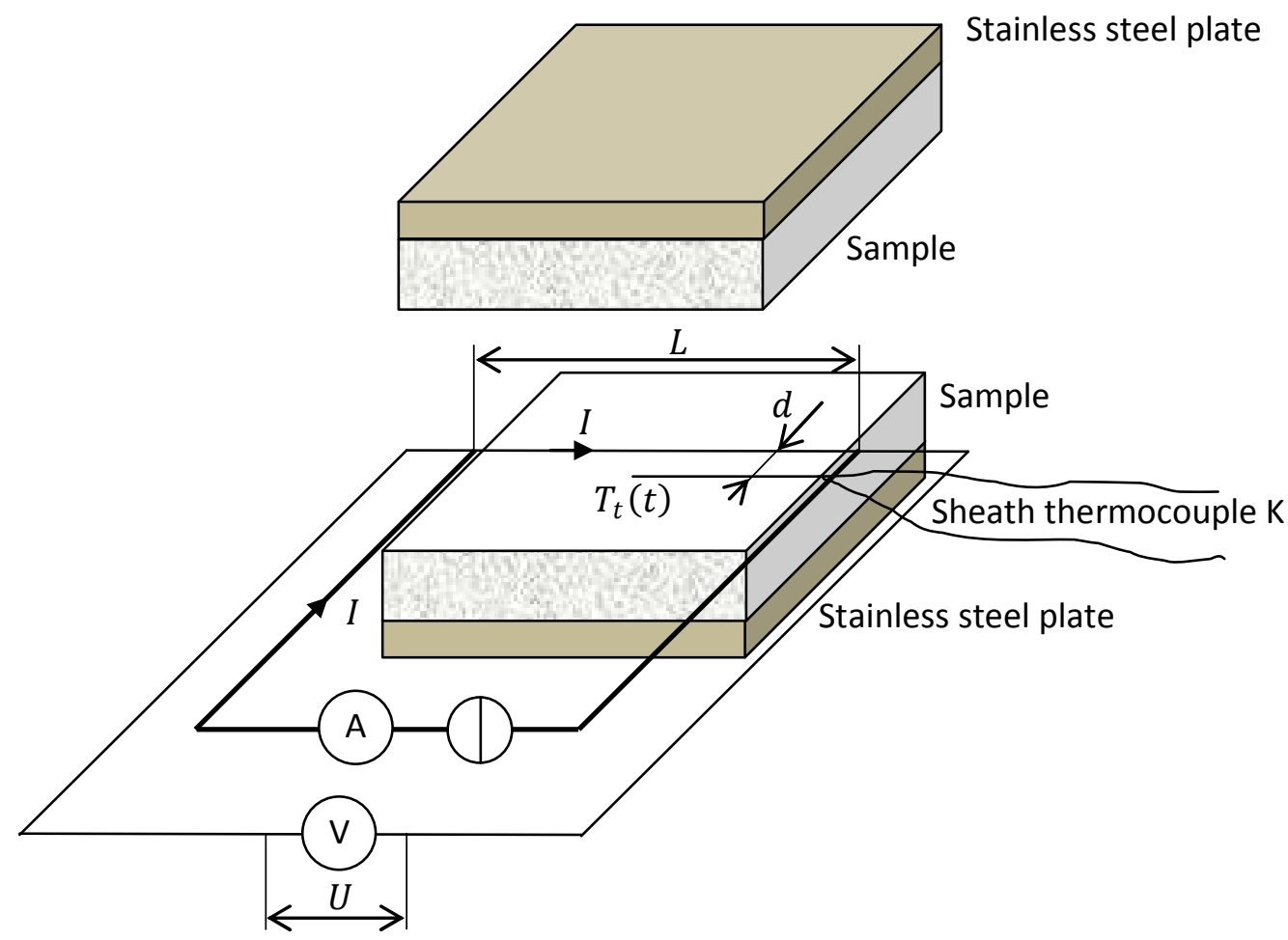

Figure 6: Schematic diagram of the experimental setup 
Moreover, the upper plate applies a pressure on the samples that minimizes the thermal contact resistances. For high temperature measurements, the device was placed in an oven Vectar equipped with a temperature controller Watlow EZ-Zone ensuring a very good temperature stability inside the oven $\left( \pm 1^{\circ} \mathrm{C}\right)$.

The temperature rise $T_{t}(t)$ is measured with a type $\mathrm{K}$ sheath thermocouple with an outer diameter of $0.5 \mathrm{~mm}$, inserted in a groove with a section $0.5 \times 0.5 \mathrm{~mm}^{2}$ realized on the halflength of the sample in order to measure the temperature at the center. The temperature is recorded with $0.1 \mathrm{~s}$ time step by a data logger Picolog TC08. The standard deviation of the temperature measurement $T_{t}(t)$ is $0.01^{\circ} \mathrm{C}$ before heating.

The heating wire made of Nickel-Chrome $80 / 20$ has a diameter of $0.5 \mathrm{~mm}$ and it is inserted in a groove with a $0.5 \times 0.5 \mathrm{~mm}^{2}$ section. The groove with the same length as the sample is realized in the middle of one face of the sample. The distance between the heating wire and the thermocouple is $d \approx 10 \mathrm{~mm}$ for the polystyrene foam and $d \approx 5 \mathrm{~mm}$ for Silcal 1100 . Two wires have been point-welded on the heating wire with a distance $L=203 \mathrm{~mm}$ for the polystyrene foam and $L=153 \mathrm{~mm}$ for Silcal 1100 between the two weldings. These wires are connected to a voltmeter Almemo 2890-9 to measure the electrical voltage $U$ between the two points. A stabilized power supply Tektronix PWS2185 produces an electrical current with a constant intensity $I$ passing through the wire. These measurements enable the calculation of the electrical resistance per meter of the heating wire by: $\frac{R_{e l}}{L}=\frac{U}{I L}$

The distance $d$ has been measured with a microscope as shown by figure 7 presenting a picture of the two grooves in one sample for the Silcal 1100 sample. Knowing that the diameter of the heating wire and of the thermocouple is $0.5 \mathrm{~mm}$, the mean distance between the two is:

$d=\frac{(5.703-0.5)+(4.131+0.5)}{2}=4.92 \mathrm{~mm}$

The relative uncertainty on $d$ is: $\frac{(5.703-0.5)-(4.131+0.5)}{4.92}=6 \%$

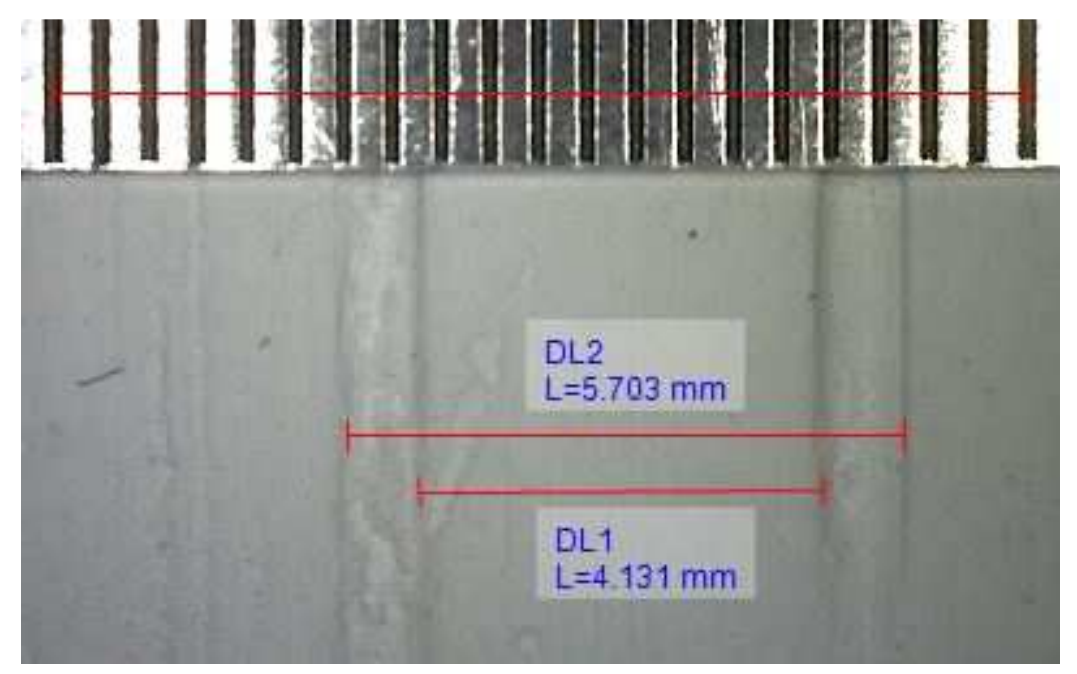

Figure 7: Picture of the grooves on the sample 
The same measurement has been realized for the polystyrene sample leading to $d=9.98 \mathrm{~mm}$ with a $5 \%$ uncertainty.

\section{Error estimation}

First, the errors caused by the uncertainty on the "known" parameters have been investigated; these parameters are: $\varphi_{w}, \rho_{w} c_{w}, r_{w}$ and $d$.

Relation (21) valid for "long times" may be written as:

$T_{t}(t)=-\frac{1}{4 \pi}\left(\frac{\varphi_{w}}{L}\right)\left(\frac{1}{\lambda_{s}}\right) E_{i}\left[\left(\frac{d^{2}}{a_{s}}\right) \frac{1}{4 t}\right]$

From this relation, one can deduce that:

- A $1 \%$ error on the length $L$ or on the heat flow rate $\varphi_{w}$ leads to a $1 \%$ error on $\lambda_{s}$.

- The estimated value of $\lambda_{s}$ is insensitive to the values of $d, \rho_{w} c_{w}$ and $r_{w}$.

- A $1 \%$ error on $d$ leads to a $2 \%$ error on the thermal diffusivity $a_{s}$.

- The estimated value of $a_{s}$ is quite insensitive to the values of $L, \varphi_{w}, \rho_{w} c_{w}$ and $r_{w}$.

The measurement uncertainty are estimated at $0.5 \mathrm{~mm}$ on $L, 0.6 \%$ on $U$ and $0.3 \%$ on $I$.

Since $\varphi_{w}=\frac{U I}{L}$ the resulting uncertainty on $\lambda_{s}$ is thus $6.6+0.3+\frac{0.5}{153} \times 100 \%=1.2 \%$ for Silcal and $6.6+0.3+\frac{0.5}{203} \times 100 \%=1.1 \%$ for polystyrene.

The uncertainty on the estimated value of $a_{s}$ is mainly due to the uncertainty on $d$. With an uncertainty of $5 \%$ and $6 \%$ on $d$ for respectively polystyrene and Silcal, the corresponding uncertainties on $a_{s}$ are $10 \%$ and $12 \%$.

These uncertainties must be added to the errors due to the model reported in Table 3 and in Table 4, and depending on the thermal properties of the material.

The method of the parallel hot wire is thus particularly well suited to the thermal conductivity measurement. On the contrary, this method only gives an approximate value of the thermal diffusivity since it is practically quite difficult to obtain a precise value of $d$.

\section{Results and discussion}

\section{Extruded polystyrene}

Table 5 presents the estimated values of the thermal conductivity $\lambda_{s}$ and of the volume heat capacity $\rho_{s} c_{s}$ obtained for the three measurements. As predicted by the theoretical study:

- The model M2 leads to the best estimation of $\lambda_{s}(+3.4 \%)$ since the model M1 leads to underestimate the thermal conductivity $(-7.7 \%)$.

- The model M2 also leads to a better estimation of $\rho_{s} c_{s}(+10 \%)$ than the model M1 $(+32 \%)$, both models overestimate $\rho_{s} c_{s}$. 
Table 5: Thermal conductivity $\lambda_{s}\left(\mathrm{~W} \mathrm{~m}^{-1} \mathrm{~K}^{-1}\right)$ and volume heat capacity $\rho_{s} c_{s}\left(\mathrm{~J} \mathrm{~m}^{-3} \mathrm{~K}^{-1}\right)$ of extruded polystyrene estimated between 0 and $300 \mathrm{~s}$ at $30^{\circ} \mathrm{C}$ with the different models

\begin{tabular}{|c|c|c|c|c|c|c|c|}
\hline & \multicolumn{4}{|c|}{$\lambda_{s}\left(\mathrm{~W} \mathrm{~m}^{-1} \mathrm{~K}^{-1}\right)$} & \multicolumn{3}{|c|}{$10^{-4} \times \rho_{s} c_{s}\left(\mathrm{~J} \mathrm{~m}^{-3} \mathrm{~K}^{-1}\right)$} \\
\hline & M0 & M1 & M2 & Reference & M1 & M2 & Reference \\
\hline 1 & $0.018<\lambda<0.029$ & 0.0293 & 0.0335 & \multirow{4}{*}{0.0324} & 2.44 & 2.02 & \multirow{4}{*}{1.85} \\
\hline 2 & $0.018<\lambda<0.029$ & 0.0291 & 0.0328 & & 2.43 & 2.06 & \\
\hline 3 & $0.018<\lambda<0.028$ & 0.0290 & 0.0326 & & 2.45 & 2.07 & \\
\hline Mean & $0.018<\lambda<0.028$ & 0.0291 & 0.0330 & & 2.44 & 2.05 & \\
\hline
\end{tabular}

Figure 8 presents the thermal conductivity estimated with the standard method (model M0) as a function of the time. The graph is quite similar to figure 5a) with a great variation of the estimated value of $\lambda_{s}$ for the different measurement times. Figure 9a) presents the experimental curve and the simulated curve obtained with the M2 model and figure $9 \mathrm{~b}$ ) presents the estimation residues obtained with models M1 and M2. The estimation residuals (x10) are also plotted for each case.

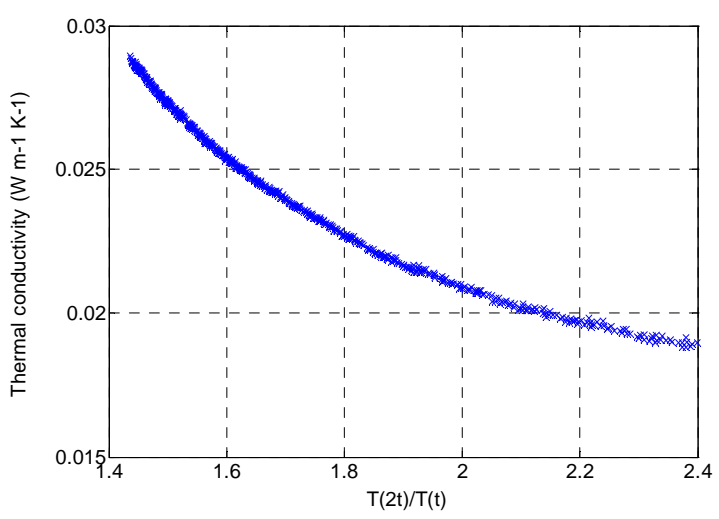

Figure 8: Estimated values of the thermal conductivity by the standard method for extruded polystyrene

a)

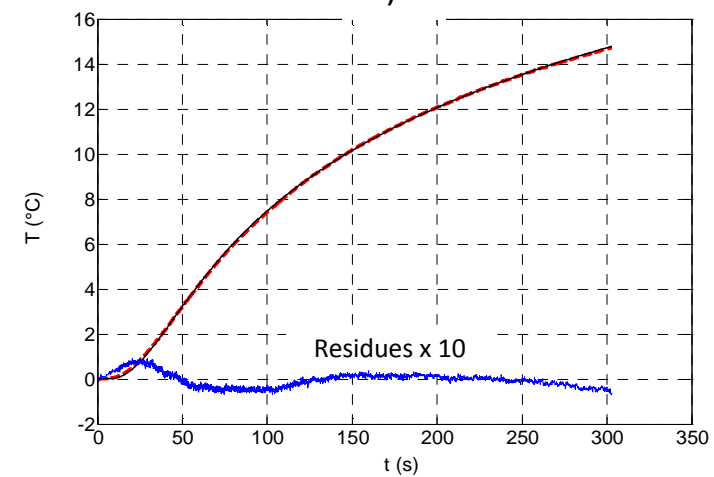

b)

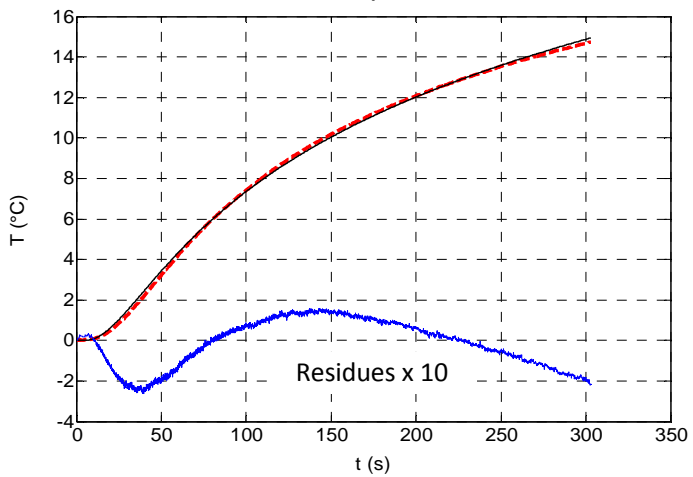

Figure 9: Experimental (--) and simulated (-) curves with estimation residues (x10): a) model M2; b) model M1

The residues obtained with the model M2 are lower than those obtained with the model M1. 


\section{Silcal 1100}

Figure 10 represents the values of the thermal conductivity estimated with the standard method for experiments realized at $200^{\circ} \mathrm{C}$ and $1000^{\circ} \mathrm{C}$, on one hand as a function of time (figures 10a) and 10c)) and on the other hand as a function of the ratio $\frac{T_{t}(2 t)}{T_{t}(t)}$ (figures 10b) and10d)).

The curves in figures 10a) and 10b) show that the horizontal step portion of the curve may be very short and sometimes even nonexistent. In these cases, the estimation with the standard method cannot be very accurate.

The curves in figures 10c) and 10d) show that it is better to use the curve representing the function $\lambda_{s}=f(t)$ rather than $\lambda_{s}=f\left[\frac{T_{t}(2 t)}{T_{t}(t)}\right]$ to plot the values of the thermal conductivity calculated by the standard method. The horizontal step portion appears more clearly on the curve $\lambda_{s}=f(t)$ enabling a better estimation of the validity limit of the semi-infinite medium hypothesis and thus of the thermal conductivity. The use of the curve $\lambda_{s}=f\left[\frac{T_{t}(2 t)}{T_{t}(t)}\right]$ requires the knowing of the value of the ratio $\frac{T_{t}(2 t)}{T_{t}(t)}$ corresponding to the limit of validity of the semiinfinite medium hypothesis. For our experimental configuration, this value is close to 1.3 since the theoretical study predicted a value between 1.4 and 1.6 (cf. figure 4).
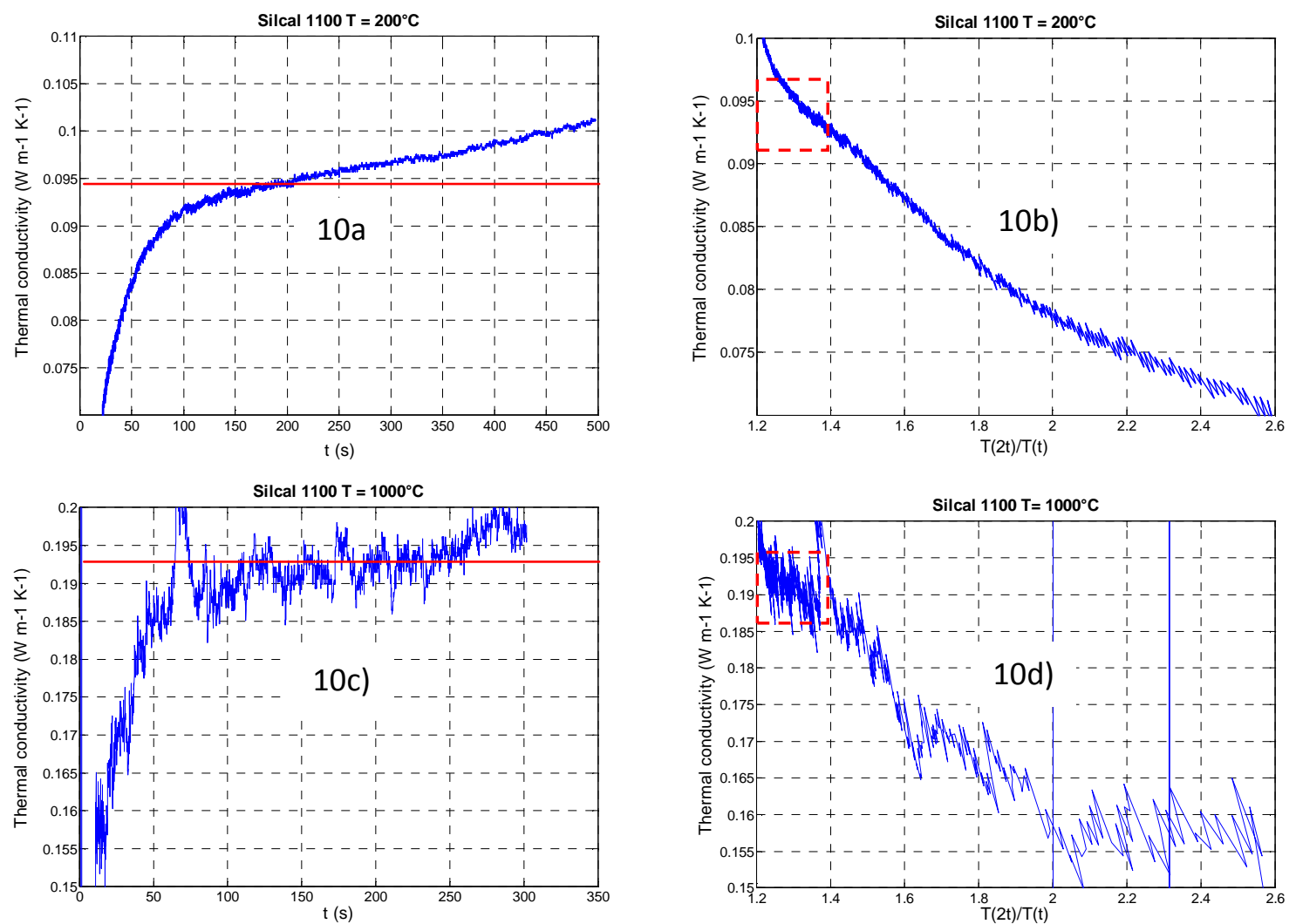

Figure 10: Estimated values of the thermal conductivity by the standard method

The curve corresponding to a temperature of $600^{\circ} \mathrm{C}$ in figure 11a) has been obtained by using the temperatures at times $t$ and $2 t$ to calculate the thermal conductivity. It indicates that the semi-infinite medium hypothesis remains valid until 400s (end of the horizontal part of the 
curve for $t=200 \mathrm{~s}$ corresponding to $2 t=400 \mathrm{~s}$ ). Figure $11 \mathrm{~b}$ ) represents the estimation residues obtained using the quadrupolar model M1 for the same experiment. The curve also shows that the semi-infinite medium hypothesis is valid until 400 s.
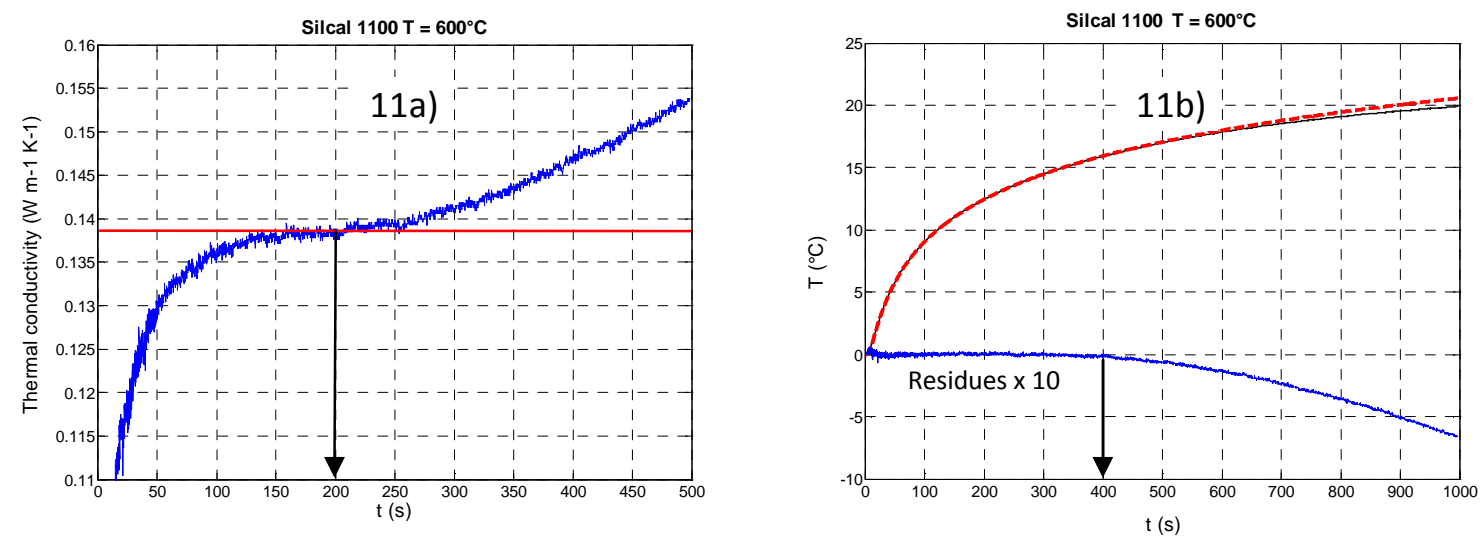

Figure 11: a) Estimated values of the thermal conductivity with the standard method; $b$ ) Experimental (--) and simulated (-) curves (quadrupolar model) with estimation residues (x10).

Figure 12 presents two examples of experimental and simulated curves obtained with the quadrupolar model $\mathrm{M} 1$ for the two extremes temperatures $200^{\circ} \mathrm{C}$ and $1000^{\circ} \mathrm{C}$. The residues are flat and centered on the null value on the estimation interval, thus the hypotheses of the model are validated. Especially the samples can be considered as optically thick.
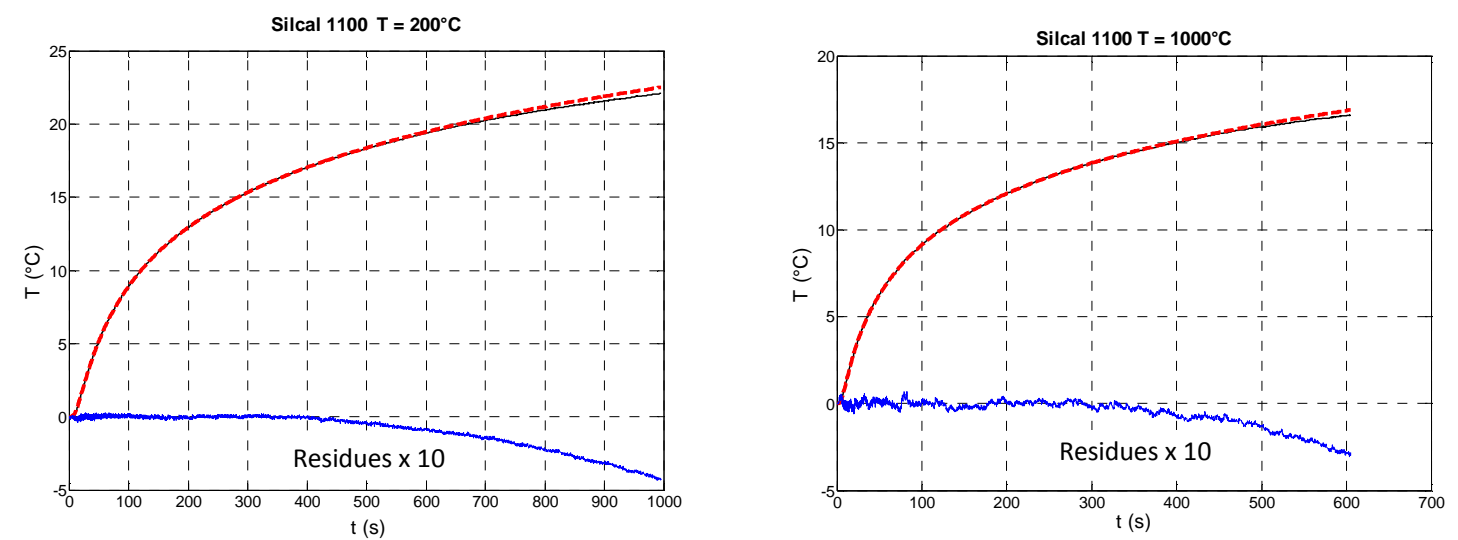

Figure 12: Experimental (--) and simulated (-) temperature + estimation residues $\times 10$ (at $200^{\circ} \mathrm{C}$ and $1000^{\circ} \mathrm{C}$ ) using model $\mathrm{Ml}$ with estimation on $300 \mathrm{~s}$.

Table 6 presents the estimation results for the 3 experiments realized for each of the 5 temperatures varying between $200^{\circ} \mathrm{C}$ and $1000^{\circ} \mathrm{C}$. The values estimated with the quadrupolar model M1 are in very good agreement (deviation lower than 2\%) with the references values presented by Ebert and Hamberger [20] as shown by figure 13. The deviations between the values calculated by the standard method and the reference values are higher (but still acceptable: lower than 5\%). The values calculated by the standard method are always slightly lower than those calculated by the quadrupolar model as predicted by the theoretical study. 


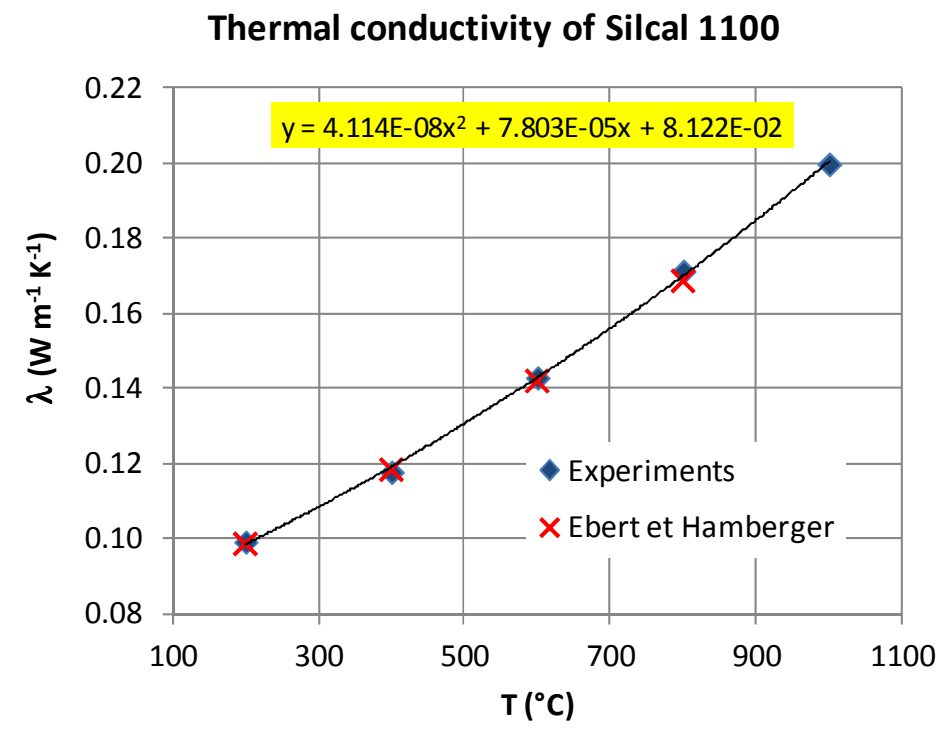

Figure 13: Thermal conductivity of Silcal 1100: estimated values by the parallel hot wire and values published by par Ebert and Hamberger [20].

Table 6: Estimated values of the parameters for all the experiments

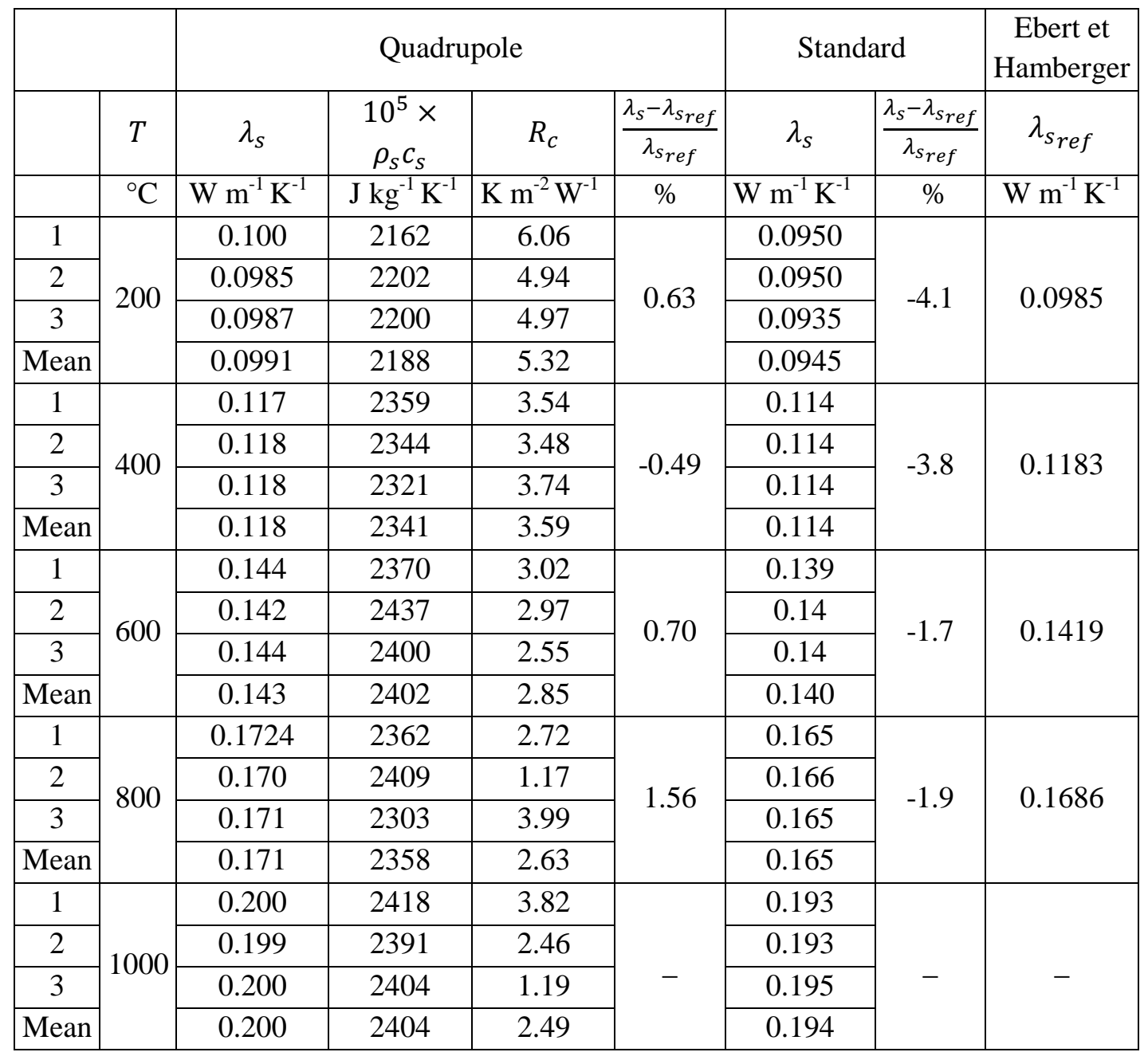


The uncertainty on $d$ is $6 \%$ thus the uncertainty on the values of the volume heat capacity $\rho c$ estimated by the parallel hot wire method (cf. Table 6) is $12 \%$. The density estimated by weighing and measurement of the dimensions of the samples is $245 \mathrm{~kg} \mathrm{~m}^{-3}$. Ohmura et al [23] measured the specific heat $c\left(\mathrm{~J} \mathrm{~kg}^{-1} \mathrm{~K}^{-1}\right)$ of the calcium silicate by drop calorimetry and they proposed the following relation valid between $100^{\circ} \mathrm{C}$ and $1000^{\circ} \mathrm{C}$ :

$c=439+82.9 \times \ln (T+273.15)$

Where $T$ is the temperature $\left({ }^{\circ} \mathrm{C}\right)$

Figure 14 represents the values of the volume heat capacity $\rho_{s} c_{s}$ estimated for each temperature:

- From parallel hot wire measurements

- using measurements of the density and relation (28) for the specific heat calculation.

The maximum deviation between the values obtained by the two methods is $6 \%$ that is quite satisfying considering the uncertainty on the value of $d$.

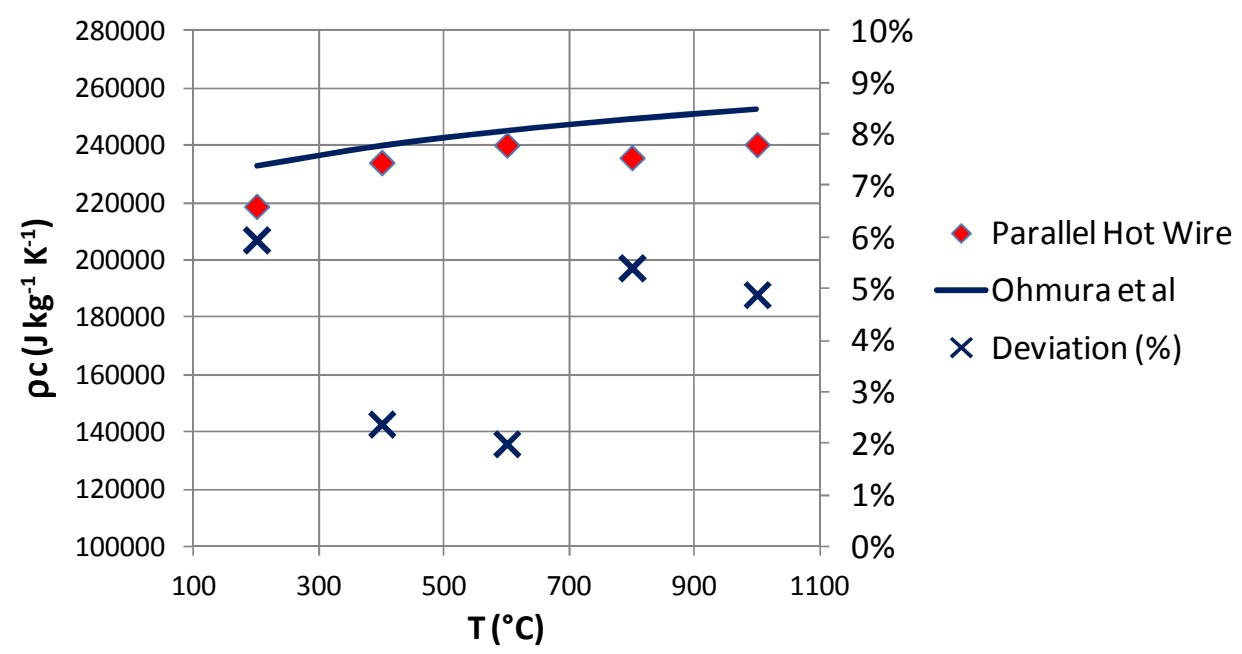

Figure 14: Values of the volume heat capacity estimated by the parallel hot wire method and given by Ohmura et al [23].

\section{Conclusion}

The estimation of the thermal conductivity $\lambda$ from a parallel hot wire experiment is mainly realized using the method described in the standard ISO 8894-2. It is based on a simplified model in which the hot wire and the thermocouple are not taken into account. First, we have proposed a polynomial expression of the function $E_{i}(u)=f\left[\frac{E_{i}(u / 2)}{E_{i}(u)}\right]$ that simplifies the estimation process of $\lambda$. Then, it has been shown that the estimation of $\lambda$ realized for $\frac{T_{t}(2 t)}{T_{t}(t)}=$ 1.5 (corresponding to "long times") is more accurate than taking the mean of all the values calculated for $\frac{T_{t}(2 t)}{T_{t}(t)}$ varying from 1.5 to 2.4 , as recommended by the standard. Nevertheless, the thermal conductivity is always underestimated, especially for the lower values. 
Then, the heat transfer in a parallel hot wire device has been modeled using a new quadrupolar model. The use of the model M2 to estimate the thermal conductivity and the volume heat capacity presents the following advantages:

- It takes into account the hot wire, the thermocouple and the thermal contact resistances between the hot wire and the sample on one hand and between the thermocouple and the sample on the other hand.

- The estimation of the thermal conductivity $\lambda$ is more precise than with the standard ISO 8894-2.

- It makes possible the estimation of lower values of the thermal conductivity $\lambda$ than with the standard method.

- The limit of validity of the semi-infinite medium hypothesis can be determined easily by analyzing the curve of the residues.

- It enables the estimation of an approximate value of the volume heat capacity $\rho c$.

It is also remarkable that the estimation of the thermal conductivity does not depend on the value of the radius of the heating wire and on the value of the distance between the heating wire and the thermocouple; this is a key advantage knowing that it is difficult to measure them accurately.

Nevertheless, it only takes into account the conduction heat transfer so that it is not suited to the estimation of thermal conductivity of semi-transparent materials. For these materials and especially at high temperature, a coupled radiation-conduction model should be used. 


\section{References}

[1] Refractory Materials- Determination of thermal conductivity- Part 2: Hot-wire method (parallel), ISO 8894-2:2007.

[2] Sengupta, K., Das, R., and Banerjee, G., "Measurement of Thermal Conductivity of Refractory Bricks by the Nonsteady State Hot-Wire Method Using Differential Platinum Resistance Thermometry," Journal of Testing and Evaluation, 20 (6) (1992) 454-459.

[3] Zhang Y.Z., Cheng S.X., Lee J.A., Ge X.S., Simultaneous measurement of thermal conductivity and thermal diffusivity of solids by the parallel-wire method, International Journal of Thermophysics, 12 (3) (1991) 577-584.

[4] Dos Santos W. N., Gregorio R., Hot-wire parallel technique: a new method for simultaneous determination of thermal properties of polymers, Journal of Applied Polymers, 85 (2002) 1779-1786.

[5] Bristow K.L., Measurement of thermal properties and water content of unsaturated sandy soil using dual-probe heat pulse probes, Agricultural and Forest Meteorology, 89 (1998) 7584.

[6] Hu R., Ma A., Wang Y., Transient hot wire measures thermophysical properties of organic foam thermal insulation material, Experimental Thermal and Fluid Science, 98 (2018) 674-682.

[7] B. Håkansson, P. Andersson, and G. Bäckström, Improved hot-wire procedure for thermophysical measurements under pressure, Review of Scientific Instruments, 59 (1988) 2269.

[8] Pettersson S., Theoretical considerations on the hot wire method applied to solids under pressure, Review of Scientific Instruments, 61 (1990) 1308-1313.

[9] Grazzini G., Balocco C., Lucia U., Measuring thermal properties with the parallel wire method: a comparison of mathematical models, International Journal of Heat and Mass Transfer, 39 (10) (1996) 2009-2013.

[10] Dos Santos W.N., Gregorio R., Numerical and experimental determination of the minimum and maximum measuring times for the hot wire parallel technique, Ceramica, 49 (2003) 29-35.

[11] De Sylos Cintra J., Dos Santos W. N., Numerical analysis of sample dimensions in hot wire thermal conductivity measurements, Journal of European Ceramic Society, 20 (2000) 1871-1875.

[12] Chen Q.H., Pang L., Wu L., Nawrat S., Liu Z., Measuring solid material's thermophysical properties with non-stationary hot wire method, Journal of Ceramic Processing Research, 16 (6) (2015) 705-711. 
[13] Maillet D., André S., Batsale J.C., Degiovanni A., Moyne C. Thermal Quadrupoles: Solving the Heat Equation through Integral Transforms. New York: John Wiley and Sons, 2012.

[14] Carslaw H.S., Jaeger J.C., Conduction of heat in solids, Oxford University Press, London, 1959.

[15] De Hoog FR. An improved method for numerical inversion of Laplace transforms. Soc. Ind. Appl. Math. 3 (3) (1982) 357-66.

[16] Marquart D., An algorithm for least squares estimation of non-linear parameters. Soc. Ind. Appl. Math., 11 (1963) 431-41.

[17] Jannot Y., Degiovanni A., Thermal properties measurement of materials, ISTE \& Wiley Editions, ISBN 978-1-78630-255-7, (2018).

[18] https://fr.wikipedia.org/wiki/Nichrome, (accessed 19 Febbruary 2019).

[19] https://www.quartz.saint-gobain.com/sites/imdf.quartz.com/files/quartzel_felts_76011.pdf, (accessed 19 Febbruary 2019).

[20] Ebert H.P., Hemberger F., Intercomparison of thermal conductivity measurements on a calcium silicate insulation material, International Journal of Thermal Sciences, 50 (2011) 1838-1844.

[21] https://www.final-materials.com/fr/45-silicate-de-calcium, (accessed 19 Febbruary 2019).

[22] https://www.ceramicsrefractories.saint-gobain.com/sites/imdf.hpr.com/files/norfoamceramic-foam-en-1056-bro.pdf, (accessed 19 Febbruary 2019).

[23] Ohmura T., Tsuboi M., Onodera M., Tomimura T., Specific heat measurement of high temperature thermal insulations by drop calorimeter method, International Journal of Thermophysics, 24 (2) (2003) 559-575. 


\section{Nomenclature}

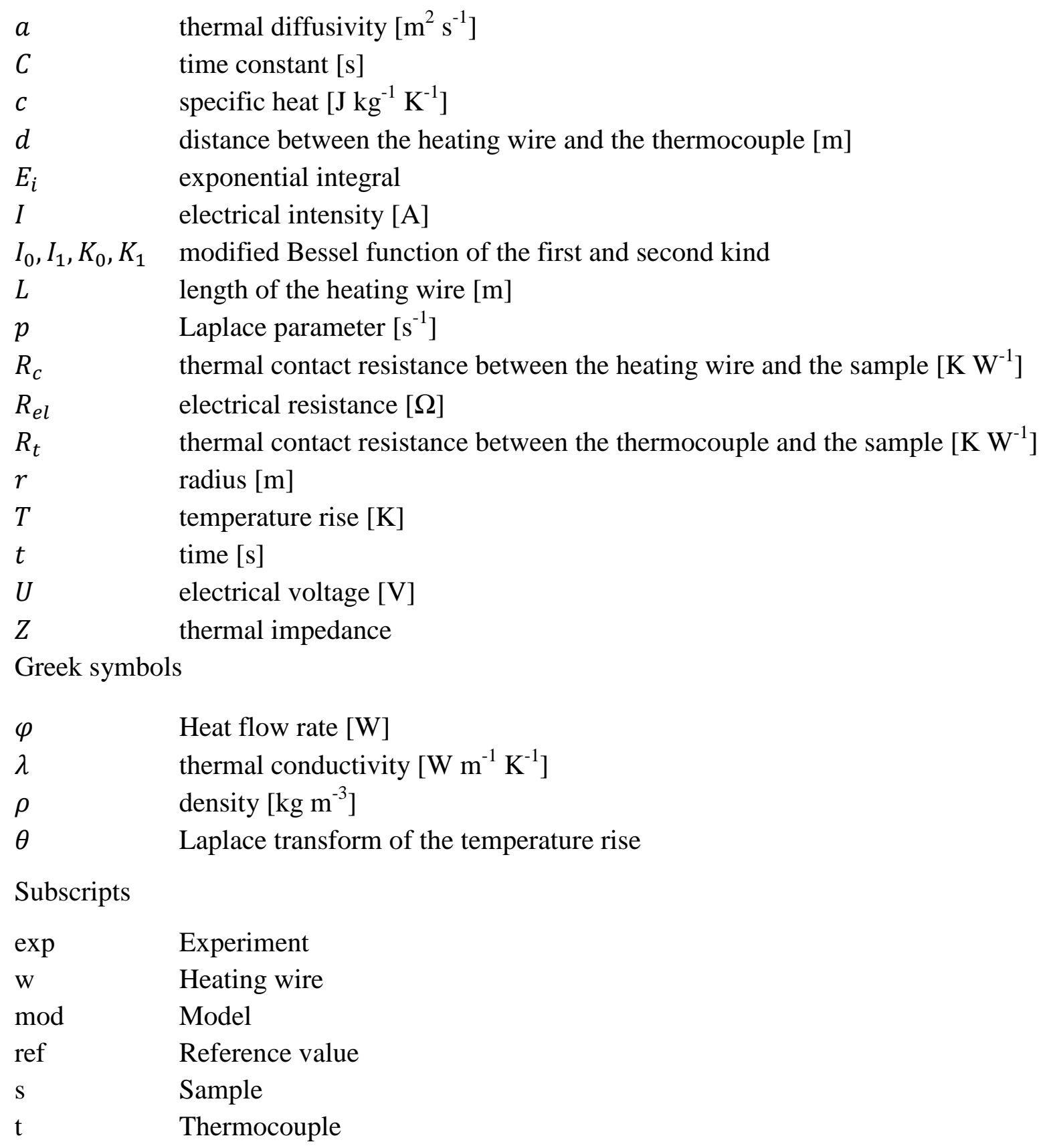

